Oral

\title{
Factors Regulating Nitric Oxide Production in Piper Sarmentosum Aqueous Extract Treated Spontaneous Hypertensive Rats
}

\author{
Maizura Mohd Zainudin ${ }^{1}$, Taher $\mathrm{F}$ T Elshami ${ }^{1}$, Hidayatul Radziah Ismawi ${ }^{1}$, \\ Fatimatuzzhara Hashim Fauzy ${ }^{1}$ \\ ${ }^{1}$ Department of Basic Medical Science, Kulliyyah of Medicine, International Islamic \\ University Malaysia
}

Presenter: Maizura Mohd Zainudin

Introduction: High blood pressure is a major risk factor for cardiovascular diseases which is one of the leading causes of death worldwide. Piper sarmentosum (PS) leaves have been widely used in traditional medicine and it has been proven for its antihypertensive and antioxidant effects. This study aims to evaluate the antihypertensive potential of PS aqueous extract (PSAE) and to investigate the factors modulating nitric oxide (NO) production through its anti-oxidant activities. Materials and Methods: PS leaves were extracted with distilled water and freezedried. The PSAE was examined to quantify their antioxidant activities through DPPH and FRAP test. It is also screened for total phenolic and flavonoids content. The antihypertensive effect of PSAE in SHR was evaluated using four different groups $(\mathrm{n}=6)$; $\mathrm{C}$ group (negative control), $\mathrm{K}$ group (PSAE $500 \mathrm{mg} / \mathrm{kg}$ ), $\mathrm{P}$ group (3 $\mathrm{mg} / \mathrm{kg}$ perindopril) and $M$ group (PSAE $+1.5 \mathrm{mg} / \mathrm{kg}$ perindopril). PSAE and other treatment were given via oral gavage for 28 consecutive days. The blood pressure and heart rate were determined using the non-invasive blood pressure monitoring (NIBPM) tail cuff technique and recorded weekly for four weeks. SHR's blood was collected for the determination of serum NO level using Griess assay. Asymmetric dimethylarginine (ADMA) and arginine levels were determined using HPLC. Results: PSAE shows good in-vitro antioxidant activities with significant reduction of BP and decreased serum ADMA level. Conclusion: PSAE enhances the clearance of ADMA that maintains arginine level, thus increasing the serum NO level, which ameliorate the blood pressure of SHR. 